\title{
(3) Fixed Duration of Venetoclax-Rituximab in Relapsed/Refractory Chronic Lymphocytic Leukemia Eradicates Minimal Residual Disease and Prolongs Survival: Post-Treatment Follow-Up of the MURANO Phase III Study
}

Arnon P. Kater, MD ${ }^{1}$; John F. Seymour, MD²; Peter Hillmen, MD³; Barbara Eichhorst, MD; Anton W. Langerak, PhD ${ }^{5}$; Carolyn Owen, MD Maria Verdugo, MD; Jenny Wu, MS ${ }^{8}$; Elizabeth A. Punnoose, PhD ${ }^{8}$; Yanwen Jiang, PhD ${ }^{8}$; Jue Wang, PhD ${ }^{8}$; Michelle Boyer, PhD ${ }^{9}$; Kathryn Humphrey, BSc $^{9}$; Mehrdad Mobasher, MD ${ }^{8}$; and Thomas J. Kipps, MD ${ }^{10}$

PURPOSE The MURANO study demonstrated significant progression-free survival (PFS) benefit for fixedduration venetoclax-rituximab compared with bendamustine-rituximab in relapsed/refractory chronic lymphocytic leukemia. With all patients off treatment, we report minimal residual disease (MRD) kinetics and updated outcomes.

METHODS Patients were randomly assigned to 2 years of venetoclax plus rituximab during the first six cycles, or six cycles of bendamustine-rituximab. Primary end point was PFS. Safety and peripheral blood (PB) MRD status - at cycle 4, 2 to 3 months after end of combination therapy (EOCT), and every 3 to 6 months thereafter-were secondary end points.

RESULTS Of 194 patients, 174 (90\%) completed the venetoclax-rituximab phase and 130 (67\%) completed 2 years of venetoclax. With a median follow-up of 36 months, PFS and overall survival remain superior to bendamustine-rituximab (hazard ratio, 0.16 [95\% Cl, 0.12 to 0.23$]$; and hazard ratio, 0.50 [95\% $\mathrm{Cl}, 0.30$ to 0.85], respectively). Patients who received venetoclax-rituximab achieved a higher rate of PB undetectable MRD (uMRD; less than $10^{-4}$ ) at EOCT (62\% v13\%) with superiority sustained through month 24 (end of therapy). Overall, UMRD status at EOCT predicted longer PFS. Among those with detectable MRD, low-level MRD $\left(10^{-4}\right.$ to less than $\left.10^{-2}\right)$ predicted improved PFS compared with high-level MRD $\left(10^{-2}\right.$ or greater $)$. At a median of 9.9 months (range, 1.4 to 22.5 months) after completing fixed-duration venetoclax-rituximab, overall only $12 \%$ (16 of 130) of patients developed disease progression (11 high-level MRD, three low-level MRD). At the end of therapy, $70 \%$ and $98 \%$ of patients with UMRD remained in UMRD and without disease progression,

ASSOCIATED CONTENT

Appendix Data

Data Supplements

Author affiliations

and support

information (if

applicable) appear at the end of this

article.

Accepted on

November 13, 2018

and published at jco.

org on December 3,

2018: DOI https://doi. org/10.1200/JC0.18.

01580

Processed as a Rapid Communication

manuscript.

Clinical trial

information:

NCT02005471.

respectively.

CONCLUSION With all patients having finished treatment, continued benefit was observed for venetoclaxrituximab compared with bendamustine-rituximab. UMRD rates were durable and predicted longer PFS, which establishes the impact of PB MRD on the benefit of fixed-duration, venetoclax-containing treatment. Low conversion to detectable MRD and sustained PFS after completion of 2 years of venetoclax-rituximab demonstrate the feasibility of this regimen.

J Clin Oncol 37:1-9. ๑ 2018 by American Society of Clinical Oncology

\section{INTRODUCTION}

Chronic lymphocytic leukemia (CLL) is a malignancy of mature $\mathrm{CD} 5^{+} \mathrm{B}$ cells. ${ }^{1}$ Treatment options for relapsed/ refractory $(R / R)$ disease have evolved from rituximab monotherapy or chemoimmunotherapy (CIT), including bendamustine-rituximab, ${ }^{2}$ to such novel agents as the kinase inhibitors ibrutinib ${ }^{3}$ or idelalisib, ${ }^{4}$ resulting in improved progression-free survival (PFS) and overall survival (OS). However, these novel therapies require continuous treatment to disease progression, which can be problematic as a result of patient intolerance, ${ }^{5}$ the high cost of therapy, ${ }^{6}$ and potential for selecting resistant subclones. ${ }^{7}$ There is therefore a need for efficacious fixed-duration targeted treatments.

Venetoclax is a highly selective inhibitor of B-cell lymphoma 2, an antiapoptotic protein that is constitutively overexpressed in CLL. ${ }^{8,9}$ Venetoclax acts independently of functional $\mathrm{TP}^{10} \mathrm{~S}^{10}$ and has proven efficacy in patients with heavily pretreated CLL. ${ }^{11,12}$

Deep remissions, including eradication of minimal residual disease (MRD) in blood or marrow, are desirable therapeutic goals in patients with $\mathrm{CLL}$, as 
attainment of undetectable MRD (UMRD) after CIT is associated with prolonged PFS and OS. ${ }^{13-18}$ Venetoclax combined with rituximab, followed by continuous venetoclax monotherapy, can result in such deep remissions ${ }^{19}$ and is therefore the first novel agent that might allow for a fixed-duration regimen. Whether MRD levels predict clinical outcome with such a regimen is currently unknown.

We compared the efficacy and safety of fixed-duration venetoclax-rituximab in R/R CLL with standard bendamustinerituximab in the phase III MURANO study. At the time of the preplanned analysis of the primary end point, the majority of patients in the venetoclax-rituximab arm were still receiving venetoclax monotherapy. Analysis revealed that venetoclax-rituximab-treated patients had significantly superior PFS and increased rates of UMRD compared with bendamustine-rituximab-treated patients. ${ }^{20}$ With this follow-up and all patients now having completed therapy, we present analyses of clinical outcomes and the impact of MRD levels on these outcomes with fixed-duration, venetoclax-based therapy.

\section{METHODS}

\section{Study Design and Conduct}

MURANO (ClinicalTrials.gov identifier: NCT02005471) is an ongoing global, phase III, open-label, randomized study investigating the efficacy and safety of venetoclax-rituximab therapy compared with bendamustine-rituximab in patients with R/R CLL. Eligibility criteria and study design have been described previously. ${ }^{20}$ The trial was conducted in accordance with the Declaration of Helsinki and the International Conference on Harmonisation of Good Clinical Practice guidelines. $^{21,22}$ The trial protocol was approved by the ethics committee at each participating institution. All patients provided written informed consent to participate.

\section{Treatment}

Patients were randomly assigned 1:1 to receive six 28-day cycles of venetoclax-rituximab followed by venetoclax $400 \mathrm{mg}$ once per day for a total of 2 years - calculated from day 1 of cycle 1 after the venetoclax dose ramp-up_ or six cycles of standard bendamustine-rituximab, ${ }^{2}$ unless disease progression or unacceptable toxicity occurred sooner. Dosing, prophylactic measures, and monitoring were as described previously. ${ }^{20}$

\section{Assessments}

Primary efficacy end point was investigator-assessed PFS, which was defined as the time from random assignment to the first occurrence of progression, relapse, or death, whichever occurred first. Peripheral blood (PB) MRD status 2 to 3 months after the end of combination therapy (EOCT) was a secondary end point and was also assessed at cycle 4 and every 3 to 6 months after EOCT. Other end points included clinical response-OS, complete response (CR), and partial response (PR) - and safety assessments. All patients were observed for safety and efficacy every 3 to 6 months through year 3 from initiation of combination therapy. Disease status was assessed by computed tomography (CT) scan at screening, cycle 4, and the EOCT visit. During the venetoclax single-agent treatment period and at follow-up visits, response was assessed clinically. As the duration of venetoclax treatment from cycle 1 , day 1 was 2 years, the time of treatment cessation is closest to the protocol-defined visit that occurred at approximately month 24 and was therefore considered to be the end of treatment (EOT) visit.

Central analysis of serial PB MRD samples was performed using allele-specific oligonucleotide-polymerase chain reaction (ASO-PCR) and/or flow cytometry on the basis of the European Research Initiative on CLL four-color assay (details in the Data Supplement). A hierarchical algorithm was used to determine MRD status using both methodologies (Appendix Table A1, online only). MRD was considered undetectable (UMRD) if the result was less than one CLL cell in 10,000 leukocytes (MRD value less than $0.0001,10^{-4}$ ). Low-level (L-)MRD was defined as $10^{-4}$ to less than $10^{-2}$, and high-level $(\mathrm{H}-)$ MRD $10^{-2}$ or greater. Safety evaluation included the monitoring of adverse events (AEs), premature study withdrawals, deaths, and venetoclax dose delays.

\section{Statistical Analyses}

At a preplanned interim analysis, the independent data monitoring committee recommended that the study undergo full analysis because the prespecified statistical boundaries for PFS were crossed - this became the primary analysis, which was published earlier. ${ }^{20}$ There is no alpha spending allocated to the current analysis of end points, performed with one more year of follow-up after the primary analysis. All $P$ values are therefore descriptive. Kaplan-Meier estimates were used to analyze the time-toevent data. Log-rank test and Cox proportional hazards regression model—stratified by del(17p) status, risk status, and geographic region - were used to compare PFS and OS across arms. Fisher's exact test was performed to compare MRD status and clinical and cytogenetic risk factors in venetoclax-rituximab patients with and without progressive disease (PD) after EOT.

\section{RESULTS}

\section{Patients}

The MURANO study enrolled 389 patients globally-194 patients in the venetoclax-rituximab arm and 195 in the bendamustine-rituximab arm (Appendix Fig A1, online only) — with well-balanced demographic and baseline disease characteristics..$^{20}$ Current data were analyzed using a cutoff date of May 8, 2018, when all patients were off treatment. In the venetoclax-rituximab arm, 174 patients (90\%) completed the venetoclax-rituximab combination phase and 130 (65\%) completed 2 years of planned 


\section{A}

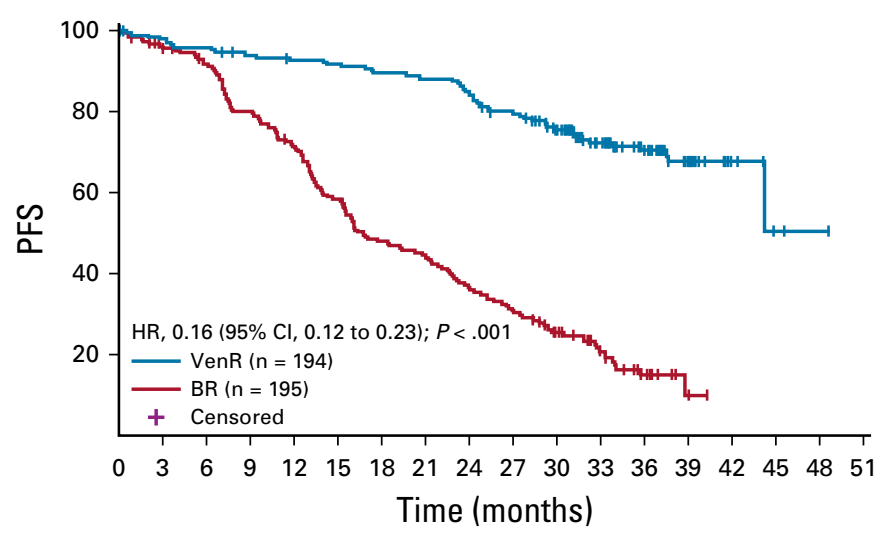

No. at risk:

$\begin{array}{llllllllllllllllll}\text { VenR } & 194 & 190 & 185 & 179 & 176 & 174 & 170 & 167 & 161 & 150 & 135 & 99 & 61 & 21 & 6 & 2 & 1\end{array}$ $\begin{array}{llllllllllllllll}\text { BR } & 195 & 178 & 164 & 142 & 128 & 103 & 84 & 79 & 65 & 55 & 41 & 26 & 10 & 2\end{array}$
B

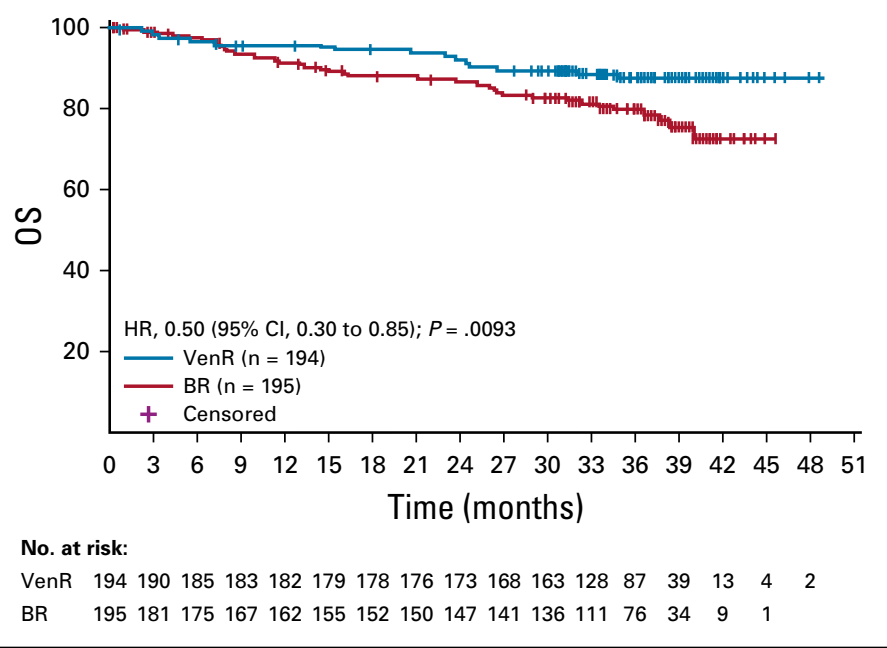

FIG 1. (A and B) Kaplan-Meier plot of (A) investigator-assessed progression-free survival (PFS) and (B) overall survival (OS) in the intention-to-treat population with 36-month median follow up. BR, bendamustine-rituximab; HR, hazard ratio; VenR, venetoclax-rituximab.

venetoclax without PD. The remainder of the patients receiving venetoclax-rituximab had PD (11\%), died without PD (1\%), or withdrew as a result of AEs (15\%; Appendix Table A2, online only) or for another reason (6\%). Characteristics of those with PD on therapy are shown in Appendix Table A3 (online only). Of those who withdrew from venetoclax treatment because of an $A E(n=32), 10$ remain free from PD; 10 had $P D$, five of whom received subsequent treatment; eight died because of the AE; and four died as a result of a different AE. Median venetoclax treatment duration from the start of ramp-up was 24.4 months (range, 0.1 to 27.9 months) and the relative dose intensity was $97.4 \%$ (range, $26 \%$ to $100 \%$; Appendix Table A4, online only).

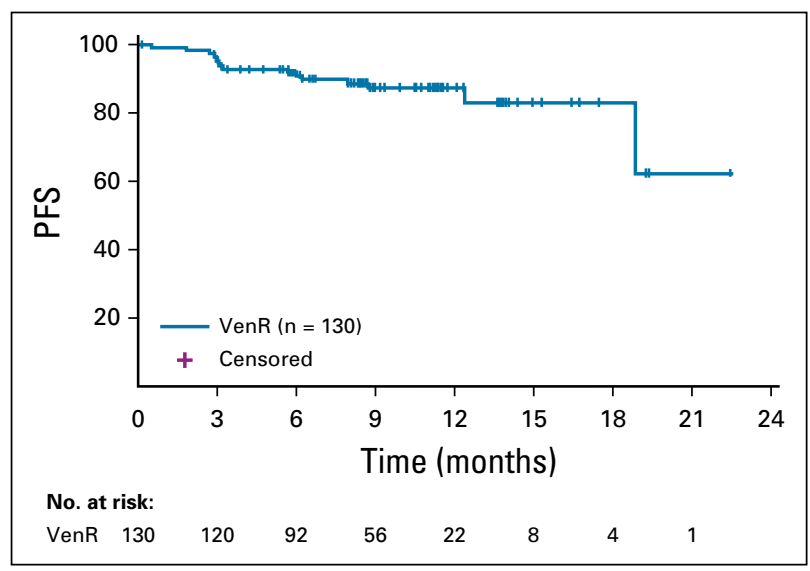

FIG 2. Kaplan-Meier plot of progression-free survival (PFS) for patients who completed 2 years of venetoclax without prior disease progression ( $n=130$; median follow-up of 9.9 months off venetoclax). VenR, venetoclax-rituximab.

\section{Efficacy}

At a median follow-up of 36.0 months, PFS with venetoclaxrituximab was superior to bendamustine-rituximab (hazard ratio [HR], 0.16 [95\% $\mathrm{Cl}, 0.12$ to 0.23]; $P<.001$; median not reached $v 17.0$ months; Fig $1 \mathrm{~A})$. Three-year PFS estimates were $71.4 \%(95 \% \mathrm{Cl}, 64.8 \%$ to $78.1 \%)$ and $15.2 \%$ $(95 \% \mathrm{Cl}, 9.1 \%$ to $21.4 \%)$, respectively. A consistent magnitude of treatment effect on PFS with venetoclaxrituximab was observed in all clinical and biologic subgroups (Appendix Fig A2, online only).

In the 130 patients who completed 2 years of venetoclax without PD, the 6- and 12-month PFS estimates from venetoclax cessation were $92 \%(95 \% \mathrm{Cl}, 87.3 \%$ to $96.8 \%)$ and $87 \%(95 \% \mathrm{Cl}, 81.1 \%$ to $93.8 \%)$, respectively (Fig 2), with a median of 9.9 months (range, 1.4 to 22.5 months) of follow-up off venetoclax. In univariate analysis, the presence of del(17p) and/or TP53 mutation and the absence of del(11q) were associated with risk of PD after venetoclax cessation (both $P<.03$; Table 1 ). However, the number of events in del(11q) patients was small $(n=1)$ and the effect diminished when Döhner hierarchical testing was applied to evaluate del(11) cases without del(17p) $(P=.25)$.

In this analysis, improvement in OS was observed with venetoclax-rituximab compared with bendamustinerituximab (HR, 0.50 [95\% Cl, 0.30 to 0.85]; $P=.0093$; 3-year estimate: $87.9 \%$ v 79.5\%; Fig 1B). Median time to next anti-CLL treatment or death was 23.9 months (range, 0.0 to 43.7 months) in the bendamustine-rituximab arm-there were too few patients at risk for this to be reliably assessed in the venetoclax-rituximab arm. Subsequent CLL-directed treatment administered after PD in 91 patients in the bendamustine-rituximab arm (Appendix Table A5, online only). Of these, 71 patients (78.0\%) 
TABLE 1. MRD Status and Clinical and Cytogenetic Risk Factors for PD in Venetoclax-Rituximab Arm Patients Alive Without Disease Progression at Month 24 ( $n=130$; univariate analysis)

\begin{tabular}{|c|c|c|}
\hline Feature (No.)* & No. (\%) With PD & $P \dagger$ \\
\hline PB MRD at EOT (month 24$) \ddagger$ & & $<.001$ \\
\hline UMRD $(n=83)$ & $2(2.4)$ & \\
\hline L-MRD ( $n=23)$ & $3(13.0)$ & \\
\hline H-MRD $(n=14)$ & $11(78.6)$ & \\
\hline Del(17p) and/or TP53 mutated & & .01 \\
\hline At least one present $(n=43)$ & $10(23.3)$ & \\
\hline Neither present $(n=78)$ & $5(6.4)$ & \\
\hline $\operatorname{Del}(11 q)$ & & .03 \\
\hline Present $(n=38)$ & $1(2.6)$ & \\
\hline Absent $(n=80)$ & $13(16.3)$ & \\
\hline Del(11q) without del(17)p§ & & .25 \\
\hline Present $(n=32)$ & $1(3.1)$ & \\
\hline Absent $(n=58)$ & $7(12.1)$ & \\
\hline IGVH status & & .14 \\
\hline Mutated $(n=38)$ & $2(5.3)$ & \\
\hline Unmutated $(n=84)$ & $13(15.5)$ & \\
\hline No. of previous therapies & & .79 \\
\hline $1(n=78)$ & $9(11.5)$ & \\
\hline$\geq 2(n=52)$ & $7(13.5)$ & \\
\hline \multicolumn{2}{|c|}{ Bulky disease (largest lymph node diameter), $\mathrm{cm}$} & 1.0 \\
\hline$<5(n=67)$ & $9(13.4)$ & \\
\hline$\geq 5(n=53)$ & $7(13.2)$ & \\
\hline Residual disease at EOCT & & .08 \\
\hline $\begin{array}{l}\text { Low nodal burden }(<1.5 \mathrm{~cm} ; \\
\qquad \mathrm{n}=64)\end{array}$ & $5(7.8)$ & \\
\hline $\begin{array}{l}\text { Intermediate nodal burden } \\
\quad(\geq 1.5 \text { to }<2 \mathrm{~cm} ; \mathrm{n}=23)\end{array}$ & $2(8.7)$ & \\
\hline $\begin{array}{l}\text { High nodal burden } \\
\qquad(\geq 2 \mathrm{~cm} ; \mathrm{n}=39)\end{array}$ & $9(23.1)$ & \\
\hline
\end{tabular}

Abbreviations: EOCT, end of combination therapy; EOT, end of therapy; $(\mathrm{H}-)$, high level; IGVH, immunoglobulin variable region heavy chain; (L-), low level; (u)MRD, (undetectable) measurable residual disease; PB, peripheral blood; PD, disease progression.

${ }^{*}$ Categories do not sum to 130 as data elements are missing for some patients. $\dagger P$ value is based on Fisher's exact test.

$\ddagger$ MRD status was not available for 10 patients at EOT.

§In Döhner hierarchical model.

received novel targeted agents, including 46 who received ibrutinib and seven who received venetoclax as next-line treatment. In the venetoclax-rituximab arm, 27 (13.9\%) of 194 patients received subsequent therapy-13 $(48.1 \%)$ of 27 patients received novel targeted agents as next treatment after PD, including eight who received ibrutinib and three who were retreated with venetoclax. Of those patients who received ibrutinib after venetoclax, two achieved PR, and efficacy data were unavailable for the remaining six patients. Among the three patients who were retreated with venetoclax, one achieved PR, one had stable disease, and one lacked follow-up efficacy data.

\section{MRD at EOCT}

MRD status assessed using ASO-PCR, and flow cytometry demonstrated high correlation $(r=.89)$ and concordance (Data Supplement and Appendix Fig A3, online only). There was also high concordance for MRD status between PB and bone marrow. Of 49 uMRD in PB, 44 (90\%) were confirmed in bone marrow in available paired samples among patients who received venetoclax-rituximab (from 47 patients; Appendix Fig A4, online only). Higher rates of PB UMRD were observed in the venetoclax-rituximab arm than in the bendamustine-rituximab arm at EOCT (Table 2) and all assessments during and after venetoclax singleagent treatment (Fig 3A). H-MRD at EOCT was less frequent with venetoclax-rituximab $(4.6 \%)$ than with bendamustine-rituximab (29.2\%; Table 2). Overall, the rate of UMRD as best MRD response at any time during the study was higher with venetoclax-rituximab (82.5\%) than with bendamustine-rituximab (23.1\%).

At EOCT, patients with UMRD had a longer duration of PFS in each treatment arm (Fig 3B) than patients with detectable MRD. Among patients with detectable MRD, those with L-MRD had a longer duration of PFS compared with patients with H-MRD for either treatment arm (venetoclaxrituximab: HR, 0.24 [95\% Cl 0.08 to 0.72 ]; bendamustinerituximab: $\mathrm{HR}, 0.22$ [95\% $\mathrm{Cl} 0.13$ to 0.38 ]). However, there were only six patients with H-MRD at EOCT in the venetoclax-rituximab arm.

Landmark analysis from EOCT demonstrated that patients in the venetoclax-rituximab arm who achieved an investigator-assessed PR with UMRD had PFS outcomes that were similar to those of patients who achieved CR with uMRD (Fig 3C; HR, 0.71 [95\% Cl, 0.24 to 2.14]). Conversely, patients who achieved PR/nodular PR with detectable MRD had inferior PFS compared with those with PR and UMRD from 18 months onwards after EOCT. Patients who achieved CR with detectable MRD-all of whom had L-MRD - had a PFS that was comparable to that of patients who achieved UMRD at this follow-up, although the number of such patients is small (HR, 1.07 [95\% $\mathrm{Cl} 0.12$ to 9.55]; Fig 3C).

\section{MRD Kinetics}

High rates of $U M R D$ and L-MRD in the venetoclax-rituximab arm at EOCT were sustained with serial assessments (Appendix Fig A5A, online only) and also found at EOT among 130 patients who completed 2 years of venetoclax (UMRD: 83 [64\%] of 130 patients; and L-MRD: 23 [18\%] of 130 patients).

At this follow-up-median of 9.9 months off venetoclaxonly two of 83 patients who were UMRD at EOT (2.4\%) developed PD. Of the patients who had detectable 
TABLE 2. MRD Status in All Patients and By Baseline Clinical and Cytogenetic Factors at EOCT

Venetoclax-Rituximab

No. UMRD (\%) L-MRD (\%) H-MRD (\%)

Factor

\begin{tabular}{|c|c|c|c|c|c|c|c|c|c|}
\hline All patientst & 194 & 62.4 & 19.1 & 4.6 & 195 & 13.3 & 23.1 & 29.2 & $<.001$ \\
\hline$<65$ & 97 & 61.9 & 15.5 & 6.2 & 89 & 12.4 & 15.7 & 31.5 & $<.001$ \\
\hline \multicolumn{10}{|l|}{ Sex } \\
\hline Male & 136 & 61.0 & 20.6 & 3.7 & 151 & 10.6 & 23.8 & 31.8 & $<.001$ \\
\hline \multicolumn{10}{|c|}{ No. of prior regimens } \\
\hline 1 & 111 & 64.0 & 21.6 & 4.5 & 117 & 12.0 & 26.5 & 31.6 & $<.001$ \\
\hline$>1$ & 83 & 60.2 & 15.7 & 4.8 & 78 & 15.4 & 17.9 & 25.6 & $<.001$ \\
\hline \multicolumn{10}{|c|}{ Bulky disease (largest lymph node diameter), $\mathrm{cm}$} \\
\hline$\leq 3.5$ & 64 & 64.1 & 18.8 & 3.1 & 59 & 10.2 & 22.0 & 30.5 & $<.001$ \\
\hline$>3.5$ & 125 & 62.4 & 17.6 & 5.6 & 127 & 15.7 & 24.4 & 29.1 & $<.001$ \\
\hline \multicolumn{10}{|l|}{ IGVH } \\
\hline Unmutated & 123 & 61.0 & 18.7 & 6.5 & 123 & 14.6 & 21.1 & 31.7 & $<.001$ \\
\hline Mutated & 53 & 64.2 & 22.6 & 1.9 & 51 & 15.7 & 27.5 & 21.6 & $<.001$ \\
\hline \multicolumn{10}{|l|}{$11 q$} \\
\hline Deleted & 61 & 65.6 & 11.5 & 4.9 & 64 & 10.9 & 26.6 & 29.7 & $<.001$ \\
\hline Not deleted & 112 & 62.5 & 24.1 & 5.4 & 105 & 16.2 & 21.0 & 28.6 & $<.001$ \\
\hline \multicolumn{10}{|c|}{ Del(17p) and/or TP53 mutated } \\
\hline
\end{tabular}

Abbreviations: EOCT, end of combination therapy; (H-), high level; IGVH, immunoglobulin variable region heavy chain; (L-), low level; (u)MRD, (undetectable) measurable residual disease; PD, progressive disease.

* $P$ value is based on Fisher's exact test.

†Data from 43 patients were missing: 14 in the venetoclax-rituximab arm and 29 in the bendamustine-rituximab arm. There were 13 PD/death/withdrawals in the venetoclax-rituximab arm and $38 \mathrm{PD} /$ death/withdrawals in the bendamustine-rituximab arm before the EOCT visit.
MRD at EOT, PD occurred in three (13\%) of 23 and 11 (79\%) of 14 patients with L-MRD and H-MRD, respectively (Fig 4). Details of the two patients in the UMRD group with PD can be found in the Data Supplement. Univariate analysis of 130 patients who completed treatment demonstrated a strong association between MRD level and progression, with UMRD being associated with better outcomes (Table 1). More patients without del(17p) and/or mutated TP53 had UMRD at EOT and remained PD free compared with those with del(17p) and/or mutated TP53 (Appendix Fig A5B, online only). The presence of minor residual adenopathy on CT scan at the EOCT visit was not predictive of achieving UMRD at EOT, nor of subsequent relapse (Table 1 and Appendix Fig A5C, online only).
Of patients with UMRD at EOT, most sustained UMRD (58 [70\%] of 83 patients; Appendix Fig A5D, online only), whereas 25 (30\%) of 83 patients developed detectable MRD. With current follow-up, this was typically L-MRD (21 [25\%] of 83 patients), all of whom remained PFS event free. Four patients who had UMRD at EOT developed H-MRD (four [5\%] of 83 patients) within a range of 6.0 to 13.4 months, one of whom subsequently developed PD, per International Workshop on CLL (iwCLL) criteria. Median time off venetoclax to the last MRD test in these 25 patients was 11.1 months (range, 5.3 to 19.3 months). Residual adenopathy on CT scan at the EOCT visit was not predictive of conversion from UMRD after drug cessation (Appendix Fig A5C). Of 25 patients who were UMRD at EOT and converted to detectable 


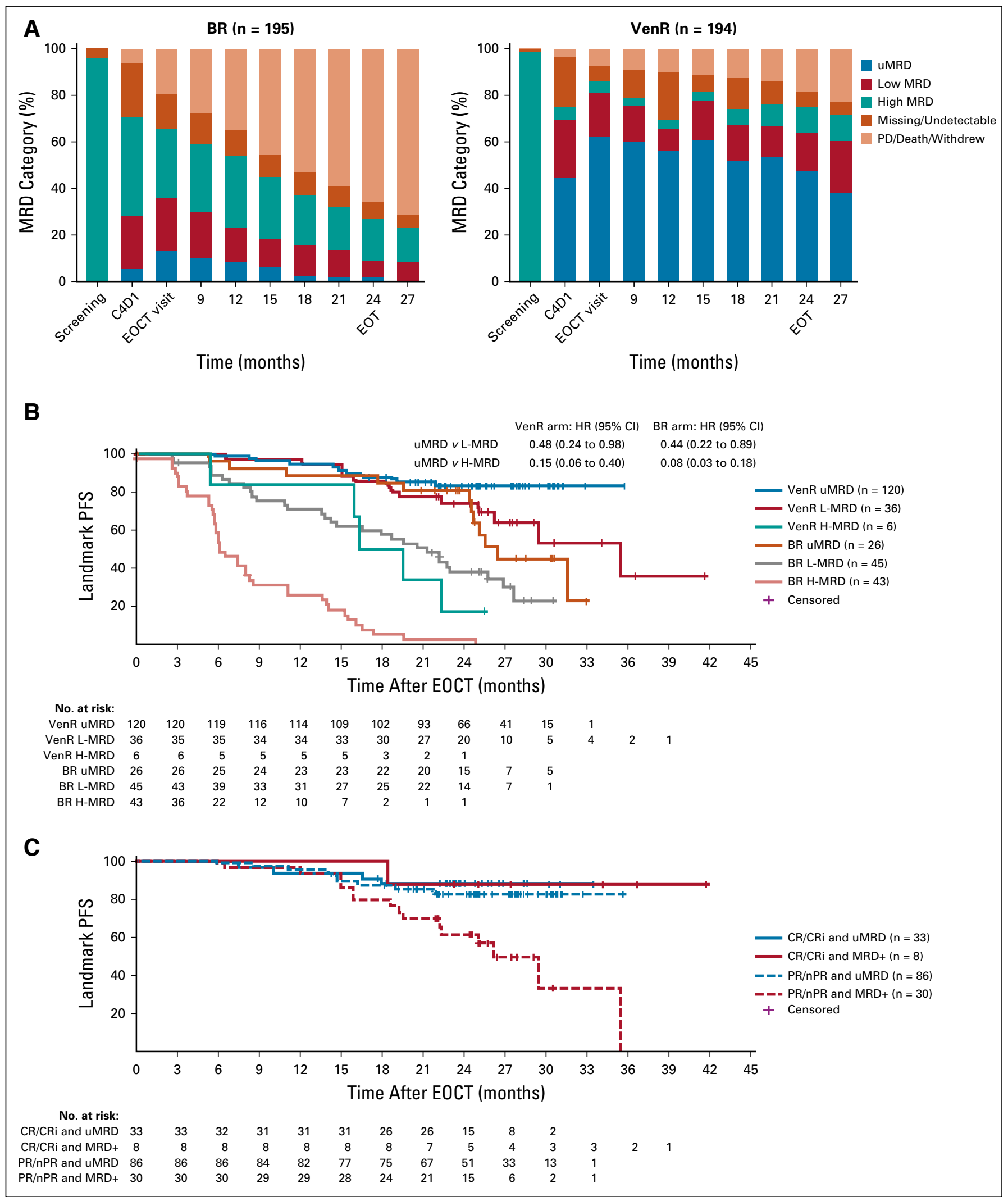

FIG 3. (A) Measurable residual disease (MRD) response over time with venetoclax-rituximab versus bendamustine-rituximab. (B) Landmark progression-free survival (PFS) analysis according to peripheral blood (PB) MRD status at end of combined therapy (EOCT) response visit (intent-to-treat [ITT] population). The analysis subset includes patients who have not experienced progression, died, or withdrawn from study before EOCT response (continued on following page) 


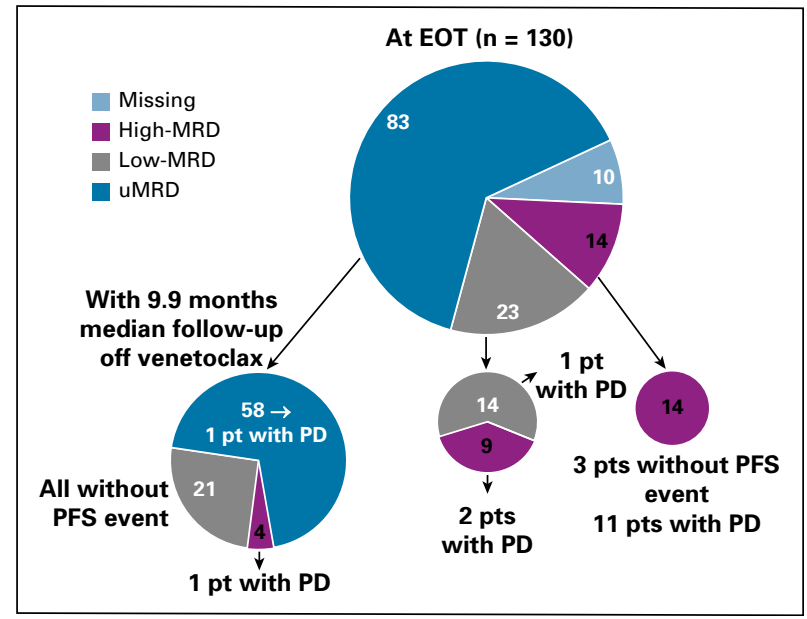

FIG 4. Measurable residual disease (MRD) changes in venetoclaxrituximab arm patients who were without a progression-free survival (PFS) event at end of treatment (EOT; $n=130$ ). (u)MRD, (undetectable) measurable residual disease; pt, disease progression; pt, patient.

MRD, 10 had residual disease at the EOCT visit with at least one lymph node having a longest perpendicular diameter of $2 \mathrm{~cm}$ or greater, three had residual disease with at least one lymph node $1.5 \mathrm{~cm}$ or greater to less than $2 \mathrm{~cm}$ at EOCT, 11 had no residual disease at EOCT, and one patient had no CT scan.

\section{Safety}

An overview of safety data for the MURANO trial during the combination and single-agent periods is provided in the Data Supplement (Appendix Table A2).

\section{Data Access}

Qualified researchers may request access to individual patient-level data through the clinical study data request platform (www.clinicalstudydatarequest.com). Additional details on Roche's criteria for eligible studies are available here: https://clinicalstudydatarequest.com/Study-Sponsors/ Study-Sponsors-Roche.aspx. For additional details on Roche's Global Policy on the Sharing of Clinical Information and how to request access to related clinical study documents, see the following: https://www.roche.com/ research_and_development/who_we_are_how_we_work/ clinical_trials/our_commitment_to_data_sharing.htm.

\section{DISCUSSION}

Analysis of the phase III global randomized MURANO study, after a median follow-up of 3 years, demonstrates substantial clinical benefit with a fixed-duration venetoclaxrituximab treatment regimen. With all patients having completed planned treatments, and with a median followup of 9.9 months off therapy, PFS with venetoclax-rituximab was superior to bendamustine-rituximab; improvements were consistent across all subgroups. These results substantiate the previously reported findings from the MURANO trial. ${ }^{20}$ Incidence of PD after the completion of venetoclax was modest at $12 \%$. Improved OS with venetoclax-rituximab was observed, despite a high rate of use of novel targeted agents in patients with PD in the bendamustine-rituximab arm. With longer follow-up, no new safety signals were identified, single-agent venetoclax after combination was tolerable, and the majority of patients completed planned treatment.

Higher rates of UMRD with venetoclax-rituximab were achieved at the EOCT response visit and as best MRD response compared with bendamustine-rituximab therapy. ${ }^{20}$ The high UMRD response rate was found in all molecular and clinical subsets, including patients with high-risk features, such as del(17p) and/or TP53 mutation. Residual H-MRD was less frequent in the venetoclaxrituximab arm than in the bendamustine-rituximab arm. Patients in each treatment arm with UMRD in PB at the EOCT response visit demonstrated prolonged PFS relative to patients who had detectable MRD. Prolonged PFS was also observed within the group with persistent MRD for those with L-MRD versus those with H-MRD.

Similar to observations with CIT in the first-line setting, eradication of detectable MRD at EOCT using venetoclaxrituximab was predictive of favorable outcome, including in patients with $\mathrm{CR}$ and $\mathrm{PR} /$ nodular PR. ${ }^{13-18}$ Longer follow-up is needed to determine the impact of PB MRD status among patients achieving $\mathrm{CR}$ or $\mathrm{CR}$ with incomplete hematologic recovery who were treated with venetoclaxrituximab. These data highlight that UMRD is an important goal of therapy irrespective of treatment approach. These findings also confirm attainment of PB UMRD as a surrogate for PFS among patients who are treated with venetoclax-rituximab. In the venetoclax-rituximab study arm, we observed that high rates of UMRD were sustained over time and also seen at EOT among patients who completed 2 years of venetoclax treatment (64\%). Of

(Continued). visit. MRD PB status derived from combining allele-specific oligonucleotide-polymerase chain reaction (ASO-PCR) and flow cytometry results. (C) Landmark PFS analysis in the venetoclax-rituximab arm according to PB MRD status and clinical response status at EOCT response visit (ITT population). The analysis subset includes patients who have not progressed, died, or withdrawn from study and have a complete response (CR), CR with incomplete hematologic recovery (CRi), nodular partial response (PR), or PR per investigator assessment at EOCT response visit. MRD PB status derived from combining ASO-PCR and flow cytometry results. BR, bendamustine-rituximab; C, cycle; CR, complete response; D, day; EOT, end of treatment; H-MRD, high-level MRD $\left(\geq 10^{-2}\right)$; HR, hazard ratio; L-MRD, low-level MRD $\left(10^{-4}\right.$ to $\left.<10^{-2}\right)$; (u)MRD, (undetectable) measurable residual disease; PD, progressive disease; ( $\left.\mathrm{n}\right) \mathrm{PR}$, (nodular) partial response; VenR, venetoclax-rituximab. 
patients in the venetoclax-rituximab arm who developed detectable MRD after EOT, most became L-MRD and remained progression free. CLL progression was only observed in two patients with confirmed UMRD status at EOT.

Overall, with a median follow-up after drug cessation of approximately 10 months, the majority of patients who were treated with venetoclax-rituximab remained free of PD by conventional iwCLL criteria-87\% at 12 months. This favorable overall outcome affirms the feasibility of fixed-duration therapy with venetoclax-rituximab in patients with R/R CLL, particularly given that either reintroduction of venetoclax or Bruton's tyrosine kinase inhibitor therapy can lead to resumed disease response in patients with progression. ${ }^{19,23}$ To evaluate a larger number of cases with venetoclax reintroduction and the likelihood of the resumption of durable disease control, as has been shown in the context of chronic myeloid leukemia after attempted TKI withdrawal, ${ }^{24}$ the MURANO protocol has been amended to prospectively collect such data. In a univariate analysis, conventional adverse pretreatment risk factors of IGVH mutational status, number of prior therapies, and disease bulk were not predictive of disease progression post-drug cessation. Presence of del(17p) and/or TP53 mutation was associated with an increased risk of clinical PD posttreatment cessation ( $P=.01$ ), but this was confounded by MRD status. At this follow-up and with just 16 PD events to date, MRD status at EOT is emerging as a strong indicator of the risk of disease recurrence and might imply that patients with H-MRD are better managed by continuing venetoclax. Residual minor adenopathy on CT assessment-here on the basis of EOCT visit, as not all patients had repeat imaging at EOT-was not associated with an increased risk of disease recurrence, which suggests that these persisting imaging findings may not represent a reservoir of viable disease. As previously reported after front-line treatment, ${ }^{17}$ serial MRD assessment may identify those patients with rising disease burden many months before clinical disease recurrence, and our data suggest that patients frequently transition through intermediate MRD levels, which does not uniformly predict imminent relapse by iwCLL criteria. Longer observation of this cohort will be needed to define the spectrum of CLL doubling times and potentially predict the timing of clinical recurrence, which will allow for therapy reintroduction before patients reach high tumor burden status. Even if a proportion of patients are ultimately destined to develop PD by iwCLL criteria and require such retreatment as venetoclax reintroduction, a number of years off drug would have quality-of-life, toxicity, and societal economic benefit. This would also minimize clonal selection pressure, relevant with other targeted therapies. ${ }^{5-7}$

This longer follow-up confirms that fixed-duration venetoclax-rituximab therapy delivers durable clinical response and a survival advantage over bendamustinerituximab therapy. Higher rates of PB UMRD at the EOCT response visit predicted prolonged benefit independent of clinical response, and patients with L-MRD also achieved better outcomes than did those with H-MRD. In the venetoclax-rituximab arm, high rates of UMRD were also sustained over time, and most of these patients remained $P D$ free after drug cessation. These data support the feasibility of a fixed duration of venetoclaxrituximab in R/R CLL. Furthermore, levels of MRD are highly predictive of duration of remission during and after venetoclax cessation.

\section{PRIOR PRESENTATION}

To be presented at the 60th ASH Annual Meeting \& Exposition, San Diego, CA, December 1-4, 2018.

\section{SUPPORT}

Supported by Genentech and AbbVie.

AUTHORS' DISCLOSURES OF POTENTIAL CONFLICTS OF INTEREST AND DATA AVAILABILITY STATEMENT

Disclosures provided by the authors and data availability statement (if applicable) are available with this article at DOI https://doi.org/10.1200/ JC0.18.01580

\section{AUTHOR CONTRIBUTIONS}

Conception and design: John F. Seymour, Peter Hillmen, Barbara Eichhorst, Elizabeth A. Punnoose, Michele Boyer, Kathryn Humphrey, Mehrdad Mobasher

Administrative support: Mehrdad Mobasher

Provision of study materials or patients: John F. Seymour, Peter Hillmen, Carolyn Owen, Michele Boyer

\section{EQUAL CONTRIBUTION}

A.P.K. and J.F.S. contributed equally to this work. 
Collection and assembly of data: John F. Seymour, Anton W. Langerak, Carolyn Owen, Jenny Wu, Elizabeth A. Punnoose, Yanwen Jiang, Michele Boyer, Kathryn Humphrey, Mehrdad Mobasher

Data analysis and interpretation: John F. Seymour, Barbara Eichhorst, Anton W. Langerak, Carolyn Owen, Maria Verdugo, Jenny Wu, Elizabeth A. Punnoose, Yanwen Jiang, Jue Wang, Michele Boyer, Kathryn

Humphrey, Mehrdad Mobasher, Thomas J. Kipps

Manuscript writing: All authors

Final approval of manuscript: All authors

Accountable for all aspects of the work: All authors

\section{ACKNOWLEDGMENT}

The authors thank the patients and their families and all MURANO study team members and investigators. Third-party medical writing assistance, under the direction of A.P.K. and J.F.S., was provided by Catherine Amey and Kate Rijnen of Gardiner-Caldwell Communications, Macclesfield, United Kingdom, and was funded by F Hoffmann-La Roche. The authors acknowledge Jill Ray for technical evaluation and implementation of the MRD assays in this study, Richa Rajwanshi for safety data interpretation, and Marcus Lefebure for help with data analysis.

The authors wish to acknowledge that Venetoclax is being developed in a collaboration between Genentech and AbbVie.

\section{REFERENCES}

1. Kipps TJ, Stevenson FK, Wu CJ, et al: Chronic lymphocytic leukaemia. Nat Rev Dis Primers 3:16096, 2017

2. Fischer K, Cramer P, Busch R, et al: Bendamustine in combination with rituximab for previously untreated patients with chronic lymphocytic leukemia: $\mathrm{A}$ multicenter phase II trial of the German Chronic Lymphocytic Leukemia Study Group. J Clin Oncol 30:3209-3216, 2012

3. Byrd JC, Brown JR, O'Brien S, et al: Ibrutinib versus ofatumumab in previously treated chronic lymphoid leukemia. N Engl J Med 371:213-223, 2014

4. Furman RR, Sharman JP, Coutre SE, et al: Idelalisib and rituximab in relapsed chronic lymphocytic leukemia. N Engl J Med 370:997-1007, 2014

5. Mato AR, Nabhan C, Thompson MC, et al: Toxicities and outcomes of 616 ibrutinib-treated patients in the United States: A real-world analysis. Haematologica 103:874-879, 2018

6. Chen Q, Jain N, Ayer T, et al: Economic burden of chronic lymphocytic leukemia in the era of oral targeted therapies in the United States. J Clin Oncol 35: $166-174,2017$

7. Landau DA, Sun C, Rosebrock D, et al: The evolutionary landscape of chronic lymphocytic leukemia treated with ibrutinib targeted therapy. Nat Commun 8: 2185, 2017

8. Souers AJ, Leverson JD, Boghaert ER, et al: ABT-199, a potent and selective BCL-2 inhibitor, achieves antitumor activity while sparing platelets. Nat Med 19: 202-208, 2013

9. Moia R, Diop F, Favini C, et al: Potential of BCL2 as a target for chronic lymphocytic leukemia treatment. Expert Rev Hematol 11:391-402, 2018

10. Anderson MA, Deng J, Seymour JF, et al: The BCL2 selective inhibitor venetoclax induces rapid onset apoptosis of CLL cells in patients via a TP53-independent mechanism. Blood 127:3215-3224, 2016

11. Stilgenbauer S, Eichhorst B, Schetelig J, et al: Venetoclax in relapsed or refractory chronic lymphocytic leukaemia with 17p deletion: A multicentre, open-label, phase 2 study. Lancet Oncol 17:768-778, 2016

12. Roberts AW, Davids MS, Pagel JM, et al: Targeting BCL2 with venetoclax in relapsed chronic lymphocytic leukemia. N Engl J Med 374:311-322, 2016

13. Böttcher S, Ritgen M, Fischer K, et al: Minimal residual disease quantification is an independent predictor of progression-free and overall survival in chronic lymphocytic leukemia: A multivariate analysis from the randomized GCLLSG CLL8 trial. J Clin Oncol 30:980-988, 2012

14. Goede V, Fischer K, Busch R, et al: Obinutuzumab plus chlorambucil in patients with CLL and coexisting conditions. N Engl J Med 370:1101-1110, 2014

15. Santacruz R, Villamor N, Aymerich M, et al: The prognostic impact of minimal residual disease in patients with chronic lymphocytic leukemia requiring first-line therapy. Haematologica 99:873-880, 2014

16. Eichhorst B, Fink AM, Bahlo J, et al: First-line chemoimmunotherapy with bendamustine and rituximab versus fludarabine, cyclophosphamide, and rituximab in patients with advanced chronic lymphocytic leukaemia (CLL10): An international, open-label, randomised, phase 3, non-inferiority trial. Lancet Oncol 17: 928-942, 2016

17. Kwok M, Rawstron AC, Varghese A, et al: Minimal residual disease is an independent predictor for 10-year survival in CLL. Blood 128:2770-2773, 2016

18. Kovacs G, Robrecht S, Fink AM, et al: Minimal residual disease assessment improves prediction of outcome in patients with chronic lymphocytic leukemia (CLL) who achieve partial response: Comprehensive analysis of two phase III studies of the German CLL study group. J Clin Oncol 34:3758-3765, 2016

19. Seymour JF, Ma S, Brander DM, et al: Venetoclax plus rituximab in relapsed or refractory chronic lymphocytic leukaemia: A phase 1b study. Lancet Oncol 18: 230-240, 2017

20. Seymour JF, Kipps TJ, Eichhorst B, et al: Venetoclax-rituximab in relapsed or refractory chronic lymphocytic leukemia. N Engl J Med 378:1107-1120, 2018

21. World Medical Association: World Medical Association Declaration of Helsinki: Ethical principles for medical research involving human subjects. JAMA 310: 2191-2194, 2013

22. International Conference on Harmonisation of Technical Requirements for Registration of Pharmaceuticals for Human Use (ICH) adopts consolidated guideline on good clinical practice in the conduct of clinical trials on medicinal products for human use. Int Dig Health Legis 48:231-234, 1997

23. Anderson MA, Tam C, Lew TE, et al: Clinicopathological features and outcomes of progression of CLL on the BCL2 inhibitor venetoclax. Blood 129:3362-3370, 2017

24. Saußele S, Richter J, Hochhaus A, et al: The concept of treatment-free remission in chronic myeloid leukemia. Leukemia 30:1638-1647, 2016 
Fixed Duration of Venetoclax-Rituximab in Relapsed/Refractory Chronic Lymphocytic Leukemia Eradicates Minimal Residual Disease and Prolongs Survival: Post-Treatment Follow-Up of the MURANO Phase III Study

The following represents disclosure information provided by authors of this manuscript. All relationships are considered compensated. Relationships are self-held unless noted. I = Immediate Family Member, Inst = My Institution. Relationships may not relate to the subject matter of this manuscript. For more information about ASCO's conflict of interest policy, please refer to www.asco.org/rwc or ascopubs.org/jco/site/ifc.

Arnon P. Kater

Honoraria: Acerta Pharma, AstraZeneca

Consulting or Advisory Role: AbbVie, Genentech, Janssen Oncology, Sunesis Pharmaceuticals

Research Funding: AbbVie, Genentech, Janssen Oncology, Roche, Celgene, Sanofi, Gilead Sciences

Travel, Accommodations, Expenses: Acerta Pharma, AstraZeneca, Genentech

John F. Seymour

Honoraria: AbbVie, Acerta Pharma, Janssen Pharmaceuticals, Roche, Sunesis Pharmaceuticals, Takeda

Consulting or Advisory Role: AbbVie, Acerta Pharma, Janssen Pharmaceuticals, Roche, Sunesis Pharmaceuticals, Takeda

Speakers' Bureau: AbbVie, Celgene, Roche

Research Funding: AbbVie, Celgene, Janssen Pharmaceuticals, Roche

Expert Testimony: Roche

Travel, Accommodations, Expenses: AbbVie, Roche

Peter Hillmen

Honoraria: Janssen, AbbVie, Gilead

Speakers' Bureau: AbbVie, Janssen, Gilead

Research Funding: Pharmacyclics (Inst), Janssen (Inst), AbbVie (Inst), Gilead (Inst), Roche (Inst)

Travel, Accommodations, Expenses: Janssen, AbbVie

Barbara Eichhorst

Honoraria: Roche, AbbVie, Gilead Sciences, Janssen-Cilag, Celgene, Novartis Consulting or Advisory Role: Gilead Sciences, Janssen-Cilag, Roche, AbbVie, Novartis

Speakers' Bureau: Genentech, Janssen-Cilag, Gilead Sciences, Celgene, AbbVie

Research Funding: Roche, AbbVie, Gilead Sciences, Janssen Pharmaceuticals Travel, Accommodations, Expenses: Roche, AbbVie, Gilead Sciences, Janssen Pharmaceuticals

Anton W. Langerak

Consulting or Advisory Role: AbbVie (Inst)

Research Funding: Genentech (Inst), Gilead Sciences (Inst)

Carolyn Owen

Honoraria: Janssen Pharmaceuticals, Teva Pharmaceuticals, Gilead Sciences, AstraZeneca

Consulting or Advisory Role: AbbVie

Research Funding: Roche Canada (Inst), Acerta Pharma (Inst), AstraZeneca (Inst), Gilead Sciences (Inst), Celgene (Inst), Janssen Pharmaceuticals (Inst)

Maria Verdugo

Employment: AbbVie

Stock and Other Ownership Interests: AbbVie

Honoraria: AbbVie

Research Funding: AbbVie

Travel, Accommodations, Expenses: AbbVie

Other Relationship: AbbVie

\section{Jenny Wu}

Employment: Genentech

Stock and Other Ownership Interests: Genentech

Travel, Accommodations, Expenses: Genentech

Elizabeth A. Punnoose

Employment: Genentech

Stock and Other Ownership Interests: Genentech

Patents, Royalties, Other Intellectual Property: Patents filed by Genentech.

Travel, Accommodations, Expenses: Genentech

Yanwen Jiang

Employment: Genentech

Stock and Other Ownership Interests: Genentech

Jue Wang

Employment: Genentech

Stock and Other Ownership Interests: Genentech

Michelle Boyer

Employment: Roche, AstraZeneca (I)

Stock and Other Ownership Interests: Roche, AstraZeneca (I)

Kathryn Humphrey

Employment: Roche

Stock and Other Ownership Interests: Roche

Mehrdad Mobasher

Employment: Genentech

Stock and Other Ownership Interests: Roche

Research Funding: Genentech

Travel, Accommodations, Expenses: Genentech

Thomas J. Kipps

Honoraria: Gilead, AbbVie, Pharmacyclics, Janssen, Verastem

Consulting or Advisory Role: AbbVie, Genentech-Roche, Gilead, Pharmacyclics, Celgene

Research Funding: AbbVie (Inst), Genentech-Roche (Inst), Oncternal (Inst), Pharmacyclics (Inst)

Travel, Accommodations, Expenses: Gilead, Janssen, Pharmacyclics, AbbVie, Genentech-Roche, Celgene

No other potential conflicts of interest were reported 


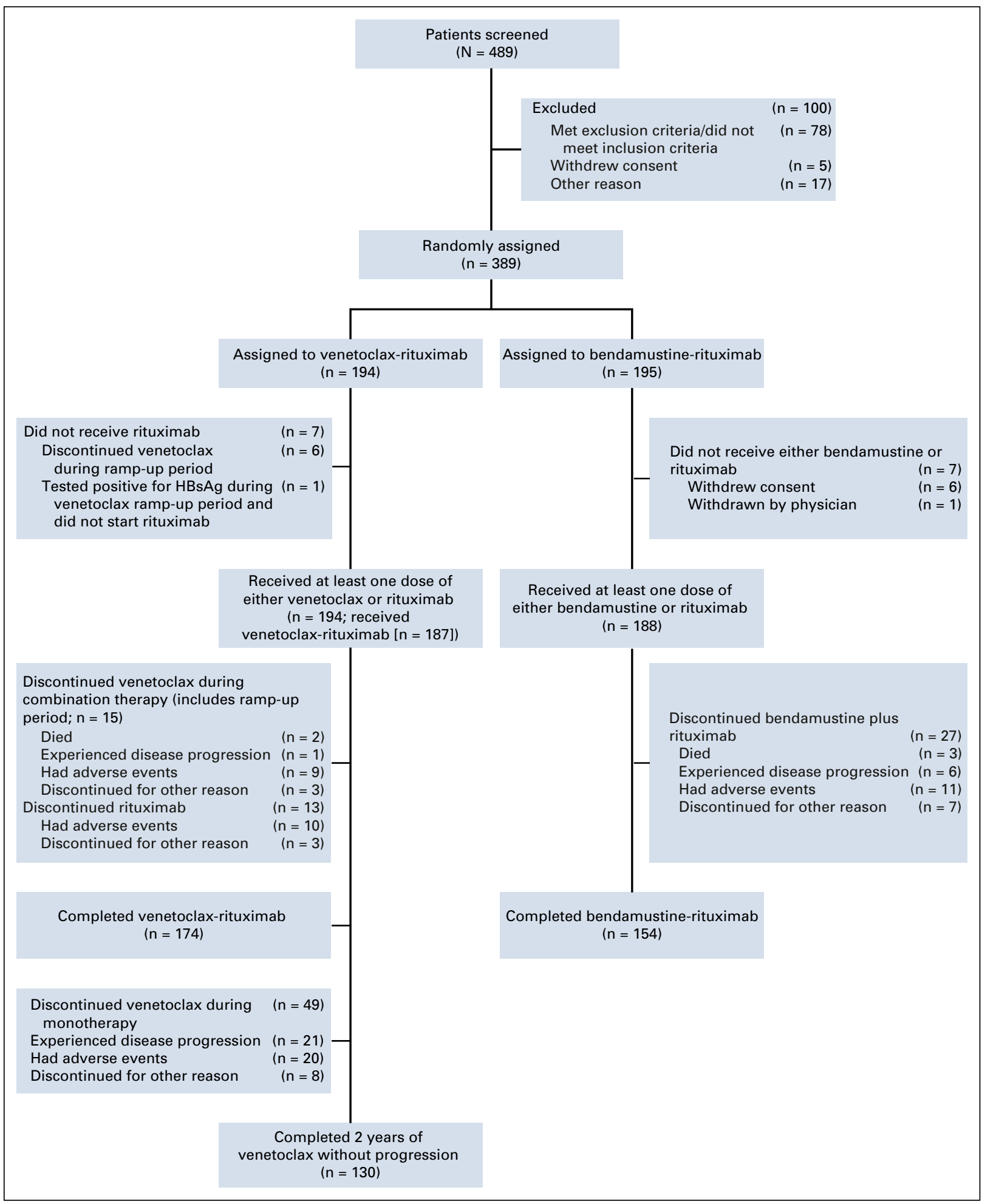

FIG A1. Patient disposition. HBsAG, hepatitis B surface antigen 


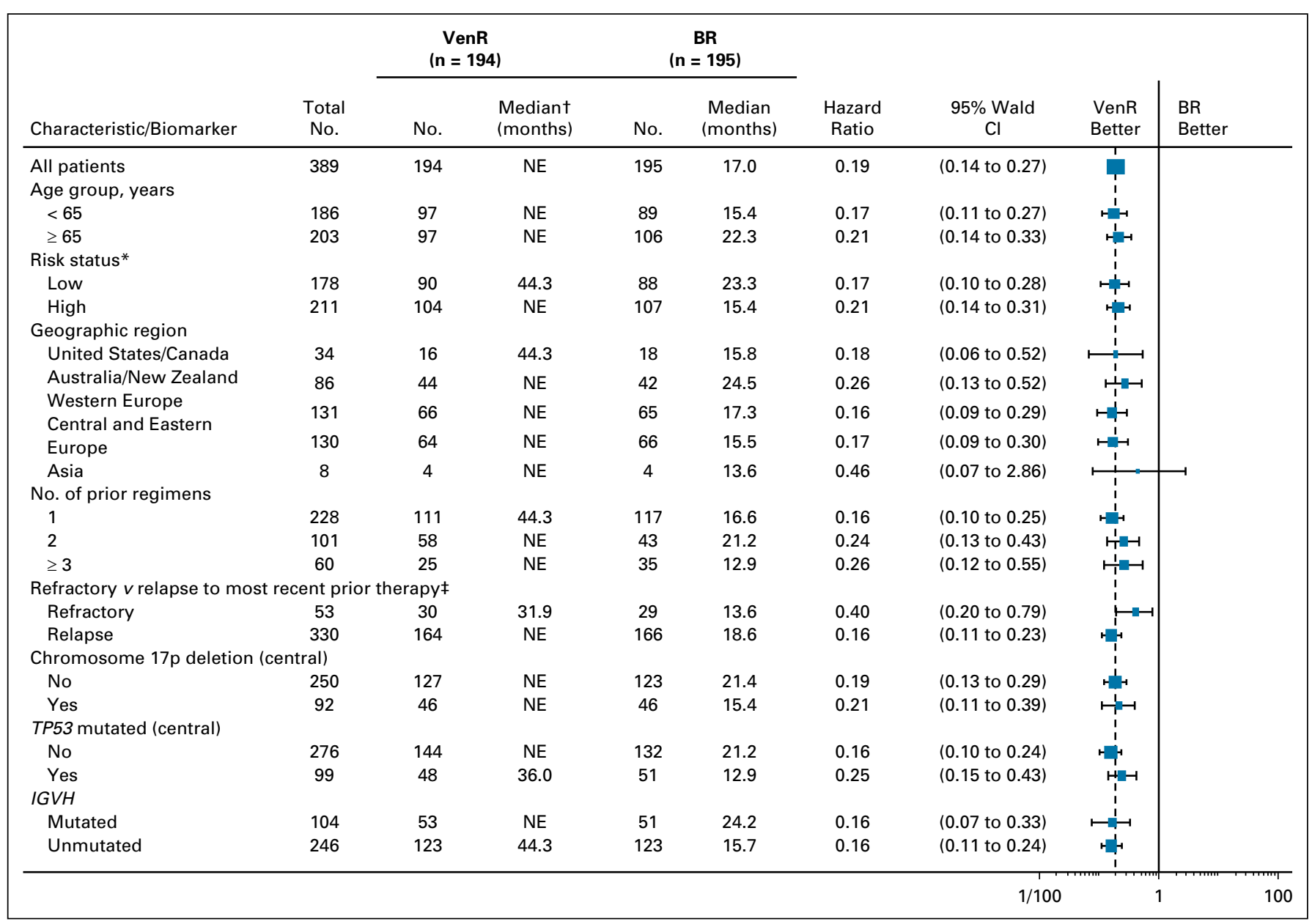

FIG A2. Prespecified subgroup analysis of investigator-assessed progression-free survival (PFS). ( ${ }^{*}$ Low risk: defined as relapse more than 12 months after chemotherapy or 24 months after chemoimmunotherapy. High risk: defined as harboring $17 p$ deletion or no response to front-line chemotherapy-containing regimen or relapsed within 12 months after chemotherapy or within 24 months after chemoimmunotherapy. ( $\dagger$ ) Medians in the VenR arm could not be reliably estimated due to too few patients at risk. ( $\ddagger)$ Defined as per iwCLL guidelines. Relapsed disease: a patient who previously achieved a complete or partial response, but after a period of 6 months or more demonstrates evidence of progression. Refractory disease: treatment failure or disease progression within 6 months of the last anti-leukemia. BR, bendamustine-rituximab; IGVH, immunoglobulin variable region heavy chain; iwCLL, International Workshop on CLL; $\mathrm{NE}$, not estimable; VenR, venetoclax-rituximab 


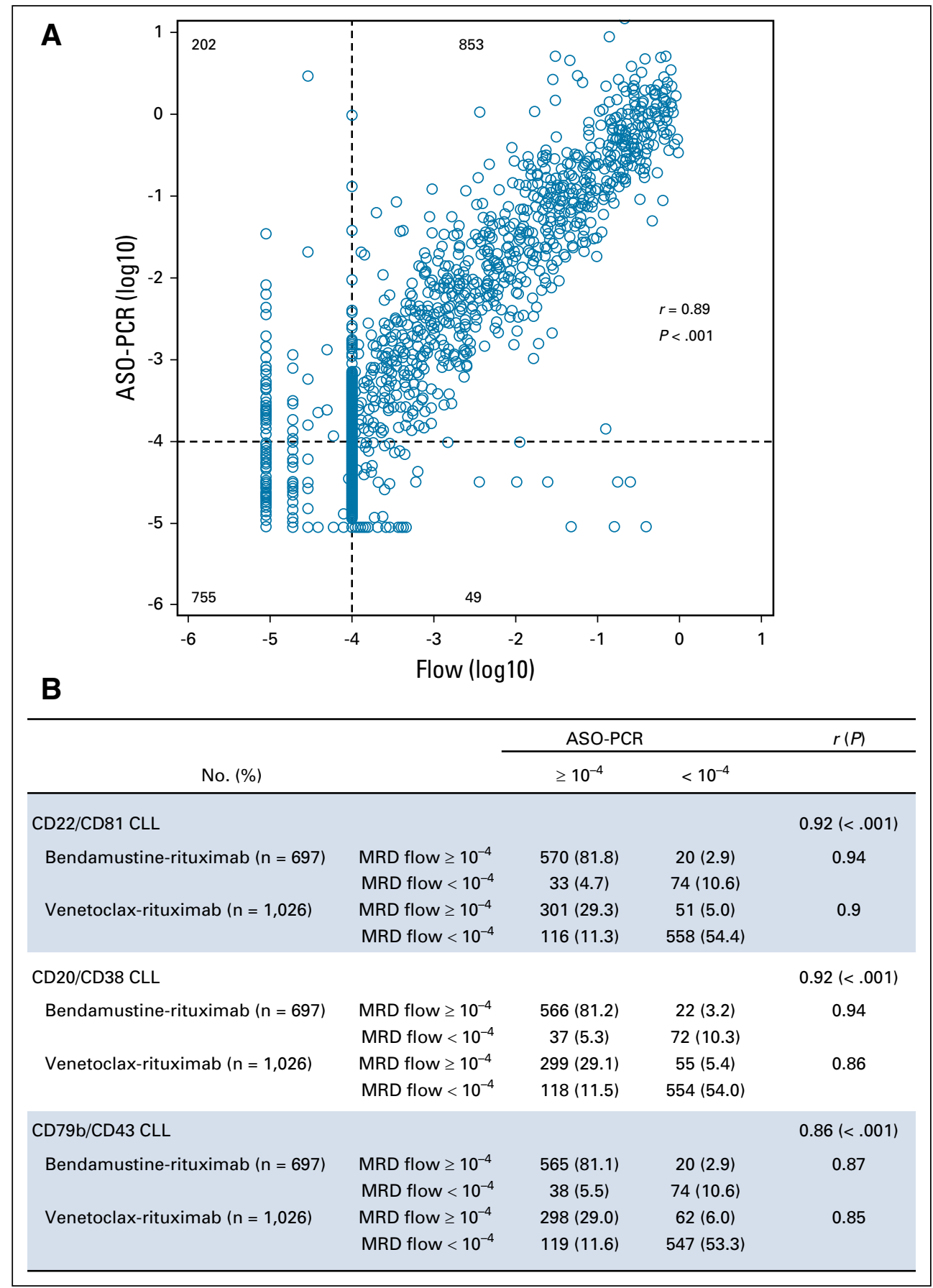

FIG A3. SO-PCR-flow concordance. (A) MRD concordance between MRD peripheral blood from flow and PCR (numerical): intent-to-treat population. (B) Correlation of quantitative MRD levels between ASO-PCR and all three antibody pairs by treatment arm. Based on data as of cut-off May 8, 2018. For MRD values below the limit of detection (LOD), LODs were represented in the graph. Correlation coefficient $r$ and $P$ value (t-test) are for positive sample only. ASO-PCR, allele-specific oligonucleotide-polymerase chain reaction; MRD, measurable residual disease. 


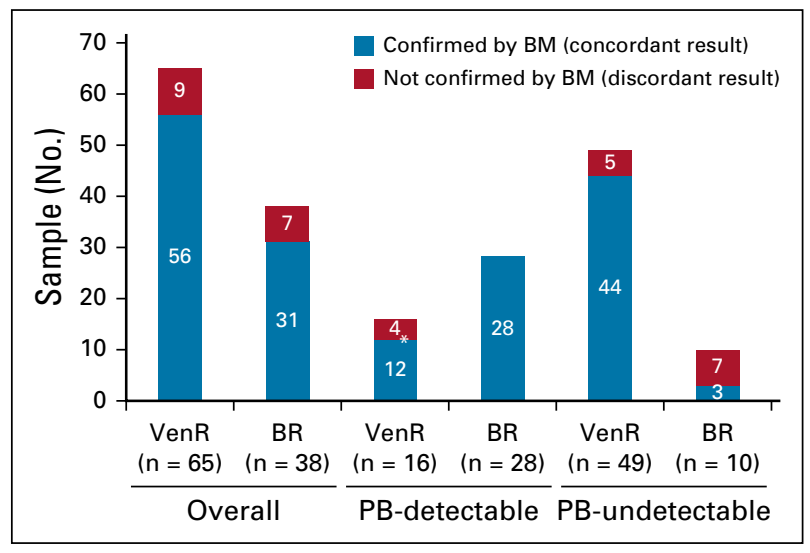

FIG A4. PB MRD is a surrogate for bone marrow (BM) MRD. * Reflects the error rate in samples with MRD levels close to the $10^{-4}$ cutoff (iwCLL recommended cutoff). BR, bendamustine-rituximab; $\mathrm{EOCT}$, end of combined therapy; EOT, end of treatment; MRD, measurable residual disease; $\mathrm{PB}$, peripheral blood; $\mathrm{BM}$, bone marrow; VenR, venetoclax-rituximab. 


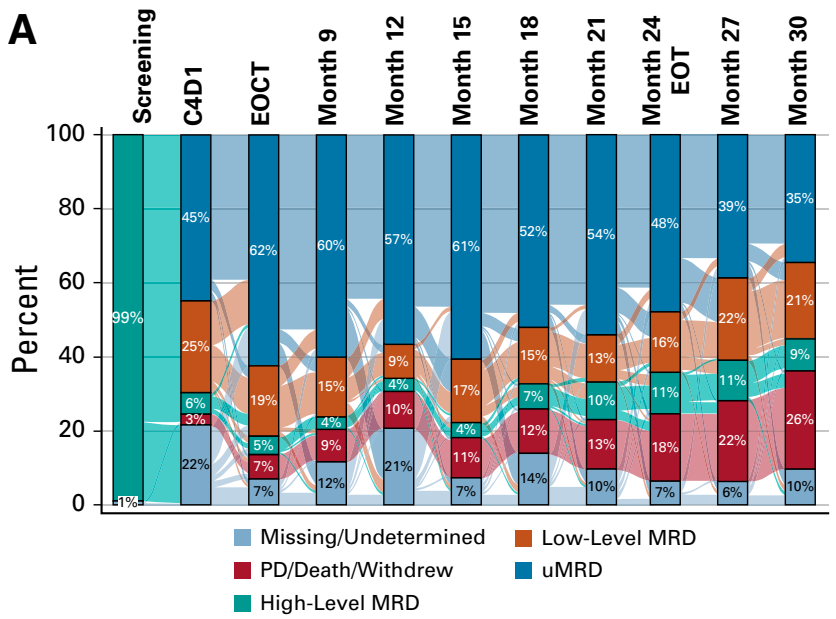

C

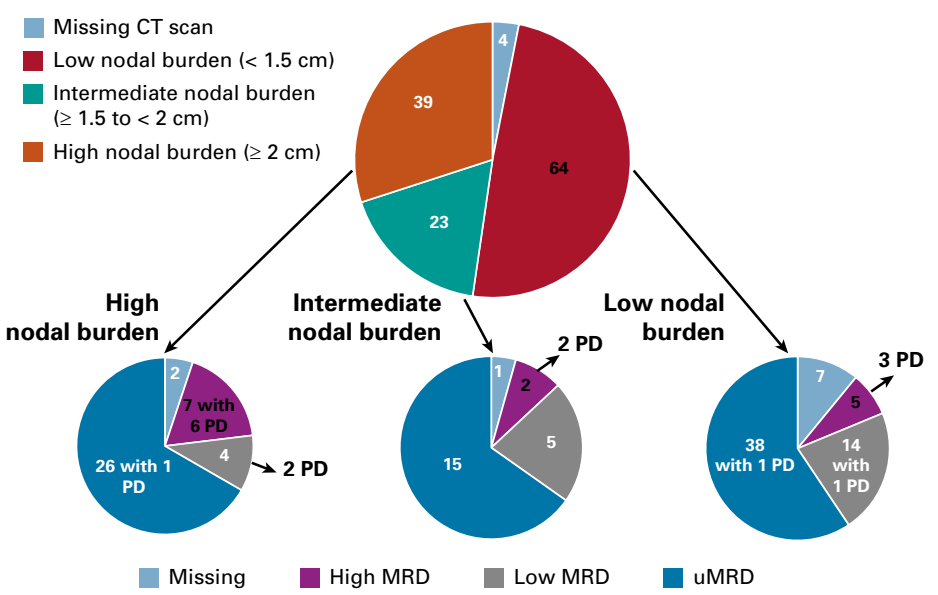

B

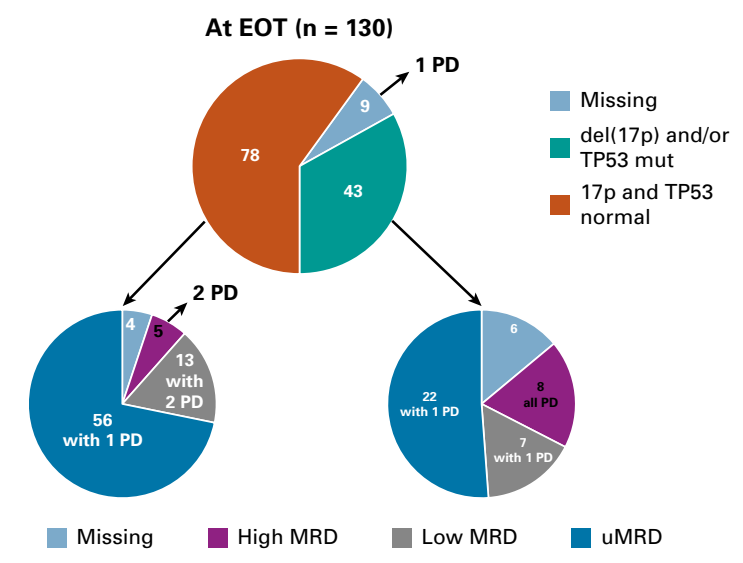

D

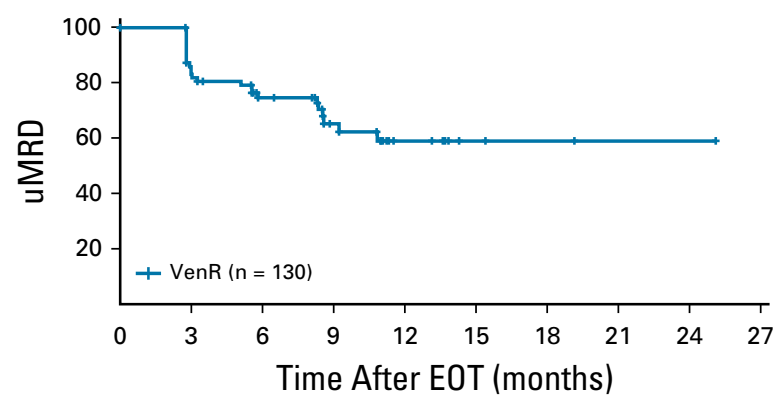

No. at risk:

$\begin{array}{llllllll}130 & 120 & 92 & 56 & 22 & 8 & 4 & 1\end{array}$

FIG A5. (A) Sankey plot is a flow diagram showing MRD kinetics in the intent-to-treat population in the venetoclax-rituximab study arm ( $\mathrm{N}=194)$ over the course of the study. The width of links between the categories within the vertical bars at each assessment time point represents the proportion of patients; the plot illustrates the dynamic change of their MRD status over time. The vertical bars are a summary of the population status at the time points labeled across the top of the chart. (B) MRD status at EOT in venetoclax-rituximab arm patients according to baseline del(17p) and/or TP53 status. (C) MRD status at EOT in venetoclax-rituximab arm patients according to residual disease status at the EOCT visit. (D) Kaplan-Meier plot of conversion to MRD+ in patients who had UMRD in peripheral blood at EOT ( $\mathrm{N}=83$ ). BM samples were not mandated in the protocol at EOT. EOT, end of treatment; PD, disease progression; ( $\mathrm{U}$ )MRD, (undetectable) measurable residual disease; VenR, venetoclax-rituximab. 
TABLE A1. Hierarchical Algorithm for Combining MRD Results from ASO-PCR and Flow Assays

Step No.

Step

\begin{tabular}{ll}
\hline 1 & Detectable MRD positive by either ASO-PCR or flow = detectable MRD \\
\hline 2 & If a sample is not detectable MRD with Step 1 and undetectable MRD by ASO-PCR and/or flow $=$ undetectable MRD \\
\hline 3 & If MRD undetermined by both ASO-PCR and flow $=$ MRD undetermined
\end{tabular}

Abbreviations: ASO-PCR, allele-specific oligonucleotide-polymerase chain reaction; MRD, measurable residual disease.

${ }^{*}$ MRD undetermined status and missing are treated as MRD positive in the intent-to-treat analysis.

TABLE A2. Overview of Safety Data for MURANO During Combination and Single-Agent Periods

Variable

Total No. of patients with $\geq 1 \mathrm{AE}$

AE leading to withdrawal from venetoclaxt

AE leading to dose reduction

AE leading to dose interruption

Grade 3-4 AEs

Fatal AEs

NOTE. Data are presented as No. (\%).

Abbreviation: $\mathrm{AE}$, adverse event.

*For the purposes of the analysis, AEs that occurred with an onset date of more than 90 days after the last rituximab dose in patients who had received at least one venetoclax dose more than 90 days after the last rituximab dose were considered to have occurred during the venetoclax single-agent treatment period. AEs that occurred with an onset date before 90 days after the last rituximab dose were considered to have occurred during the venetoclax-rituximab combination treatment period.

†There was a total of $41 \mathrm{AEs}$ that resulted in withdrawal from venetoclax therapy in 32 patients. AEs leading to withdrawal of venetoclax therapy during the combination period were neutropenia $(n=4 ; 2.1 \%)$, thrombocytopenia $(n=3 ; 1.5 \%)$, neoplasms (metastasis [unspecified], colorectal cancer $[n=2 ; 1.0 \%$ ]), febrile neutropenia $(n=1 ; 0.5 \%)$, anemia $(n=1 ; 0.5 \%)$, autoimmune hemolytic anemia $(n=1 ; 0.5 \%)$, acute respiratory failure $(n=1 ; 0.5 \%)$, appendicitis $(n=1 ; 0.5 \%)$, peritoneal tuberculosis $(n=1 ; 0.5 \%)$, pneumonia $(n=1 ; 0.5 \%)$, pyrexia $(n=1 ; 0.5 \%)$, status epilecticus $(n=1 ; 0.5 \%)$, and sudden cardiac death $(n=1 ; 0.5 \%)$. AEs leading to withdrawal of venetoclax during the single-agent period were neoplasms (colorectal cancer, metastatic malignant melanoma, pancreatic cancer, lung and peritoneal neoplasms $[n=5 ; 2.6 \%])$, neutropenia $(n=3 ; 1.5 \%)$, thrombocytopenia $(n=2 ; 1.0 \%), A L T$ increase $(n=1 ; 0.5 \%)$, ascites $(n=1 ; 0.5 \%)$, asthenia $(n=1 ; 0.5 \%)$, autoimmune hemolytic anemia $(n=1 ; 0.5 \%)$, Crohn's disease $(n=1 ; 0.5 \%)$, diarrhea $(n=1 ; 0.5 \%)$, immune thrombocytopenia purpura $(n=1 ; 0.5 \%)$, hydrothorax $(n=1 ; 0.5 \%)$, lung infection/pneumonia $(n=2 ; 1.0 \%)$, sudden death $(n=1 ; 0.5 \%)$, and vertigo $(n=1 ; 0.5 \%)$. 
TABLE A3. Characteristics of Patients $(n=25)$ Who Experienced Progression Before End of Therapy

Characteristic

Value

\begin{tabular}{lc}
\hline No. of prior lines of therapy & \\
\hline 1 & $14(56)$ \\
\hline 2 & $8(32)$ \\
\hline 3 & $3(12)$ \\
\hline Del17p & $6(24)$ \\
\hline Deleted & $16(64)$ \\
\hline Normal & $3(12)$ \\
\hline Unknown & $12(48)$ \\
\hline P53 mutation & $12(48)$ \\
\hline Mutated & $1(4)$ \\
\hline Not mutated & $9(36)$ \\
\hline Unknown & $13(52)$ \\
\hline P53 mutation and/or del17p & $3(12)$ \\
\hline No & $63(59.7 ; 28-81)$ \\
\hline Yes
\end{tabular}

NOTE. Data are presented as No. (\%), unless otherwise noted.

TABLE A4. Study Treatment Exposure in Safety-Evaluable Patients

\begin{tabular}{|c|c|c|c|c|}
\hline Variable & $\begin{array}{l}\text { Venetoclax-Rituximab } \\
\qquad(n=194)\end{array}$ & $\begin{array}{c}\text { Venetoclax-Rituximab } \\
\text { Combination Period } \\
(n=194)\end{array}$ & $\begin{array}{l}\text { Venetoclax-Rituximab Monotherapy } \\
\text { Period* }(n=187)^{*}\end{array}$ & $\begin{array}{l}\text { Bendamustine- } \\
\text { Rituximab } \\
(n=188)\end{array}$ \\
\hline \multicolumn{5}{|c|}{$\begin{array}{l}\text { Venetoclax treatment duration } \\
\text { (months) }\end{array}$} \\
\hline No. & 194 & 194 & 173 & NA \\
\hline Mean (SD) & $21.0(7.2)$ & $6.7(2.1) \dagger$ & $16.0(4.2)$ & NA \\
\hline Min-max & $0.1-27.9$ & $0.1-24.9 \dagger$ & $0.5-21.9$ & NA \\
\hline \multicolumn{5}{|c|}{ Dose intensity (\%) } \\
\hline No. & 189 & 189 & 172 & 188 \\
\hline Mean (SD) & $89.6(17.1)$ & $91.6(14.5)$ & $90.2(18.3)$ & $96.7(8.2)$ \\
\hline Median & 97.4 & 97.2 & 99.3 & 100 \\
\hline
\end{tabular}


TABLE A5. Response to Subsequent Therapy After PD in the Venetoclax-Rituximab and Bendamustine-Rituximab Arms

Patients Receiving Subsequent Therapy

\begin{tabular}{|c|c|c|c|c|c|c|}
\hline Treatment Arm & Total & CR & $\mathrm{PR}^{*}$ & SD & PD & NE/NA \\
\hline Ibrutinib monotherapy/combination & 8 & 0 & 2 & 0 & 0 & 6 \\
\hline Idelalisib monotherapy/combination & 1 & 0 & 0 & 1 & 0 & 0 \\
\hline Chemotherapy and/or immunotherapy & 14 & 1 & 2 & 2 & 3 & 6 \\
\hline \multicolumn{7}{|l|}{ Bendamustine-rituximab arm } \\
\hline Ibrutinib monotherapy/combination & 46 & 5 & 22 & 4 & 1 & 14 \\
\hline Venetoclax monotherapy & 7 & 0 & 2 & 0 & 0 & 5 \\
\hline Idelalisib monotherapy/combination & 7 & 1 & 4 & 0 & 1 & 1 \\
\hline Non-BTKi & 3 & 0 & 2 & 0 & 1 & 0 \\
\hline Other & 1 & 0 & 1 & 0 & 0 & 0 \\
\hline
\end{tabular}

NOTE. Data are given as No.

Abbreviations: BTKi, Bruton's tyrosine kinase inhibitor; CR, complete response; NA, not assessed; NE, not evaluable; PD, disease progression; PR, partial response; $\mathrm{SD}$, stable disease.

*Includes one patient who achieved a nodular partial response.

†Excludes ibrutinib. 